\title{
Research Pathway towards MAC Protocol in Enhancing Network Performance in Wireless Sensor Network (WSN)
}

\author{
Anitha K \\ Research Scholar \\ Visvesvaraya Technological University \\ Department of Computer Science \& Engineering \\ RajaRajeswari College of Engineering \\ Bangalore, India
}

\author{
Usha $S$ \\ Professor \&Head \\ Department of Computer Science Engineering \\ RajaRajeswari College of Engineering \\ Bangalore, India
}

\begin{abstract}
The applications and utility of Wireless Sensor Network (WSN) have increased its pace in making an entry to the commercial market since the last five years. It has successfully established its association with Internet-of-Things (IoT) and other reconfigurable networks. However, in this advent of exponential progress in technology, WSN still suffers from elementary problems of energy efficiency, scalability, delay, and latency where Medium Access Control (MAC) protocols hold the primary responsibility. This paper reviewed the frequently used MAC protocols and studied their advantages and limitations followed by most recently carried out implementation work towards WSN performance enhancement. The paper finally outlines the unsolved problems from the existing research work and discussed briefly the research gap followed by a chalked out plan of tentative future work to address the research gap from existing review.
\end{abstract}

Keywords-Delay; energy issues; latency; MAC protocol; scalability; Wireless Sensor Network (WSN)

\section{INTRODUCTION}

A collaboration of small sensor nodes $(\mathrm{SN})$ distributed in a region to monitor the specific atmospheric parameters is considered as wireless sensor network (WSN) [1]. An SN is an extremely delicate and little electronic device which has very limited computational capability and resource availability [2]. The WSN applications are found in e.g. warehouse to habitat monitoring system. An important thing to understand in the applications of the sensor network is that there is two types of applications e.g. time critical application and mission critical application. Time critical applications are those who have constraints of time bound, but mission critical applications have multiple constraints. The majority of the real-time applications for emergency situation call for timeliness message transmission. The problem starts shooting up when it is the dynamic network. Hence, the node dissipates maximum energy to ensure sending the data packet. Unfortunately, if the node dissipates too much energy not only its network lifetime minimizes but its communication performance also degrades significantly. Also, the majority of the other problems e.g. routing problems, security problems, and load balancing problems in WSN arises from energy issue itself. Even after the decades of the research work, this problem has not been effectively solved. In this problem, Medium Access Control or commonly known as MAC protocols are widely utilized in WSN to solve energy dissipation problems. The scheme allows a proper scheduling of a node to go to sleep and wake mode for saving energy where they are in idle state. The MAC protocols are primarily responsible for furnishing better communication channel among the sensors by sharing the access medium very precisely and fairly [3]. The protocol stack of WSN, MAC layers assist in rectifying the errors that have evolved right from the physical layer with other responsibilities too e.g. addressing, framing, error controls, solving contradiction of channel among the multiple sensors, node mobility, etc. [4]. There are MAC protocols dedicated for both single and multiple channel network structures of WSN [5]. Such schemes are essentially meant for streamlining the data transmission to a better communication system for ensuring energy efficiency and scalability [6]. It is known that inclusion of duty cycle in MAC modeling incorporates better energy modeling [7], [8]. The researches considering duty cycle was not much successful as the majority of them suffer from significant latency. This is because no data will be transmitted or received unless and until the sensors come out their sleep mode. Hence, the duty cycle is a prominent factor that directly affects the network performance of WSN. Hence, in past one decade, multiple MAC protocols have been evolved to resolve energy issue, scalability, delay, and latency. Research work on MAC protocols aged more than a decade and now it is essential to study for the objective of optimizing the system model for issues like energy optimization, time-synchronization, etc.

Therefore, this paper elaborates an extensive review of the existing research contributions, its methodologies adopted, advantages and limitations in the domain of WSN. It reviews both frequently used research work as well as recent research implementation using MAC protocols. Section II discusses the fundamental characteristics of MAC protocols in WSN followed by Taxonomies of Research Work in Section III. Section IV discusses the recently conducted research work in this direction followed by brief highlights of explored research gap in Section V. Future scope is given in Section VI followed by Conclusion in Section VII. 


\section{MAC PROTOCOLS IN WSN}

In WSN, the MAC protocols are responsible for defining the methods of accessing the resources present in a channel. This protocol is mainly meant for sharing resources over the same channels by a large number of sensors not impacting the network performance in the negative sense. The different functional requirements of MAC protocols are as follows [9]:

- Reliability: The MAC protocols ensure the error-free communication process between two nodes. It uses retransmission and acknowledgment mechanism to do so.

- Medium Access: The nodes are controlled to participate in the communication process at any instances of time.

- Framing: The MAC protocol has a precise way to represent the format of a "data frame" in achieving the specific encapsulation and appropriate operation of deencapsulation in the communication process.

- Error Control: The MAC protocols in WSN should have a higher degree of error detection capability supported by codes for correcting errors.

It is essential that an efficient MAC protocol must possess the property of minimizing latency and energy consumption with maximized throughput and fairness in as a compulsory parameters in WSN. The effective set of properties that are present in the MAC protocol in WSN are as follows:

- Collision Avoidance: MAC protocol utilized to avoid a collision. If the node is to send data, it will check first network allocation vector. If that network allocation vector value is not set to zero, then node determines that medium is busy. Hence, proper allocations of slots precisely assist the node accordingly to its sleep and awaken states.

- Energy Efficiency: This is accomplished by making use of MAC protocol. During message transmission, the message is divided into the frame and sent into the network to conserve more transmittance energy.

- Scalability and Adaptability: MAC protocol also aims to minimize significant problems of scalability as well as adaptability. This protocol helps in fine tuning at network size, node density variation along with the topology effectiveness.

- Channel Utilization: As MAC protocol is all about allocation and scheduling, hence it significantly assists in optimal utilization of channel.

- Latency: MAC protocol maintains a superior communication among the nodes by sharing their sleepwake schedules. This significantly attempts to minimize delay in WSN.

- Throughput: This is improved by making use of MAC protocol. This MAC protocol will set the amount of data transferred from sender to receiver in the unit frame by this it will improve the throughput.

- Fairness: MAC protocol attempts to maintain a better balance among all the channel parameters and thereby it encourages better fairness over the WSN even in high traffic condition.

Apart from the above characteristics of MAC protocol in WSN, the significant advantageous features of it are as follows:

- Reduce energy waste caused by idle listening in sleep schedule.

- Uses time synchronization overhead, it may prevent sleep schedule announcement.

- Sleep time is high while the collision probability is less in the MAC protocol.

- In this, low latency can be attained with traffic sources and also energy consumption in the network will be minimized.

- Ensures high throughput under low contention.

- Needs less schedule maintenance.

Similarly, the inherent MAC protocols issues in WSN are as follows:

- The transmission slot value of MAC protocol is needed to be set as high as $7 \mathrm{x}$ time than the random access period. This results in transmission and reception duty cycle to a higher value.

- Increase idle listening is draw back present in MAC protocol this is caused by listening to all slots before sending.

- Redundancy in MAC protocol which causes waste in transmission power.

- Occurrences of overhead in Synchronization due to prolonged interval of listening period MAC protocol.

Hence, the MAC protocols are associated with both advantages and disadvantages in performance. The next section discusses the taxonomies of the research work in MAC scheme, where the frequently applied MAC protocols are discussed briefly.

\section{TAXONOMIES OF RESEARCH IN MAC SCHEMES}

The MAC schemes in WSN include i.e. 1) scheduled; and 2) content-based MAC protocol. The scheduled protocols work on restricted clock synchronization demands in a WSN. The listen and transmit period is scheduled to resist the consequences of collusion, idle listening and over the hearing. They also make use of Carrier Sensed Multiple Access (CSMA) methods. The Content-based MAC (CBMAC) protocols have flexibility in time synchronization demands and are capable of automatically fine tuning itself as per the new topologies by adding up new sensors. This section will present some of the frequently known MAC protocols very briefly [10]: 


\section{A. Sensor MAC (S-MAC)}

S-MAC is contention-based and is an enhanced type of conventional IEEE 802.11 standard. S-MAC uses two-time frames where a one-time frame is utilized to listen while another frame is utilized for sleeping. SMAC also uses some commonly known beacons called as SYNC e.g. Acknowledgement (ACK), Request to Send (RTS), clear To Send (CTS). However, there is energy dissipation during the listening of SN.

\section{B. Berkley MAC (B-MAC)}

This is contention-based MAC scheme in WSN and is improved ALOHA scheme with preamble sampling. It allows faster switching of the node without any chance to miss any data. B-MAC has better energy conservation feature using long preambles and unsynchronized duty cycles. It also uses a filter mechanism for enhancing the assessment of channel and reliability. The biggest advantage of B-MAC is its capability to reduce the idle listening as well as duty cycle without using any SYNC packet.

\section{Timeout MAC (T-MAC)}

This helps in solving the uniform sleep listen period of sleep in S-MAC. In this, threshold time is used to end the period at an idle period.

\section{Wise MAC (W-MAC)}

It allows the preamble to precede every data to alert the receiver. This scheme uses acknowledgments as the schedules of sleep during the data exchange for updating their neighbor node's sleep period. Finally, it adjusts its schedule by referring the same of its neighbor node.

\section{E. Traffic Adaptive MAC Protocol (TRAMA)}

The prime aim of this scheme is to save energy using TDMA scheme. This scheme considers that MAC layer can compute the duration of transmission that data is transmitted to the application layer called as SCHEDULE_INTERVAL. It then prioritizes the highest hop neighbor as well as evaluates the slot numbers using time as [t+SCHEDULE_INTERVAL]. Finally, the announcements of the slots are done, and a bitmap is utilized to flag the defined receivers with their respective scheduled packets.

\section{F. Data Gathering MAC (D-MAC)}

It is a scheduled-based protocol aims to enhance the energy efficiency and minimize latency. The technique permits division of time into various small slots and uses acknowledgment for every time slots for receiving and transmitting a single data packet. One significant advantage of D-MAC is it has a smaller delay.

\section{G. Convergent MAC (C-MAC)}

This protocol is mainly meant for energy efficiency as well as latency by using excessive RTS message in the forwarding transmission. C-MAC also checks its channel twice for assessing the channel quality. The significant beneficial point of C-MAC is its capability to resist overhead due to synchronization as well as its ability to work at minimal duty cycles. C-MAC also uses any cast for performing packet forwarding mechanism to explore the forwarding node and perform converging with unsynchronized duty cycle.

Table 1 summarizes the technique used, significances and drawbacks of the frequently used MAC protocols in WSN. It can be observed that MAC protocols are mainly meant for energy efficiency enhancement of each SNs be it cluster head or member node in a sensor network. In spite of the presence of various MAC protocols, there are less number of MAC protocols which has received standardization tag among the research community. The prime reason behind this is that most of MAC protocols are highly dependent on applications posing a great challenge in evolving up as generalized standards.

TABLE. I. SUMMARY OF FREQUENTLY USED MAC PROTOCOLS

\begin{tabular}{|l|l|l|l|}
\hline Protocol & Technique used & Advantage & Limitation \\
\hline S-MAC & Virtual cluster, Uniform duty cycle & Low energy & Latency during broadcast \\
\hline B-MAC & Channel assessment & Minimal overhead & Degraded performance at high traffic \\
\hline T-MAC & Overhearing, duty cycle (adaptive) & Active time (adaptive) & Pre-pones sleep schedule \\
\hline Wise-MAC & Lower schedule of preamble sampling & Less overhead & Not energy efficient \\
\hline TRAMA & Schedule interval & Energy efficient & Random access period \\
\hline D-MAC & Excessive RTS message & Minimal latency & Higher rate of aggregation \\
\hline
\end{tabular}

\section{EXISTING TECHNIQUES}

This section intended to survey of existing recent techniques in MAC protocols. The work of Abdel et al. [11] described the Hybrid MAC Protocol (HMACP) for the realtime applications. The model contains both Time Division Multiple Access (TDMA) and Frequency Division Multiple Access (FDMA) that offers a smooth real-time communication among the randomly deployed WSN. This schedules the nodes communication which avoids collisions, interfaces optimization and gives less delay. In this, the simulation was performed for SNs and random sinks. The outcomes give the reliable, scalable and end to end delay.
The model proposed in Fig. 1 consists of four processing units such as Initialization, Synchronization \& Discovery, slot or frequency attribution, and medium access. For example, Each node after the deployment turns on their radio transmitter and will wait for a message. After receiving the message by every node, the discovery step will be actuated by saving the messages of the source address in the neighbor list and also broadcast the message in a proper ID. Once the direct information is collected, every node will forward their respective neighbor's list to the base station (BS). The BS will construct a graph and is connected to graph $G(V, E)$ representing a network, where $V-$ set of nodes \& E-Edges . 


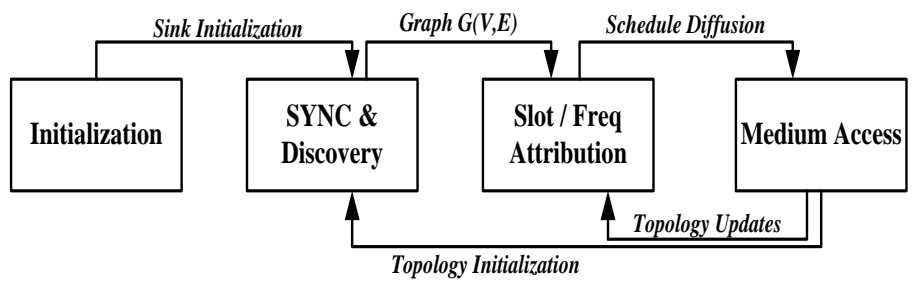

Fig. 1. Functioning process of Abdeli et al. [11] work.

The slot/frequency attribution (SFA) unit contains schedule plan construction, basing over graph (G) by the attribution of every node $m \subseteq V$, where $\mathrm{m}$ is some nodes and $V$ is set of nodes. The BS will perform the diffusion of schedule to nodes. After the above hierarchical process nodes will send the data packets.

The sampling rate (SR) effect on the collision probability is described in Dong et al. [12] especially for hybrid MAC protocols of WSN. From the investigation of the author, it is known that the collision is the main cause of energy waste in MAC protocols under the saturate condition considering the sampling rate greater than the transmission rate. Later the investigation study towards the effect of collision for WSN was performed by considering sleeping MAC protocol. Karahan et al. [13] developed an energy efficient hybrid MAC (EEHMAC) protocol for a large-scale WSN. In this work, the techniques like TDMA and CSMA are considered. The reason for the use of TDMA is that it reduces unnecessary collisions in channels while the CSMA responses rapidly for events. With this model author achieved high throughput and energy optimization.

A study was based on traffic adaptive MAC protocol with multichannel and allocation of dynamic slots in Zhuo et al. [14] for the WSN. The contribution of this work is that it presented a hybrid iQueue-MAC (TDMA and CSMA) mechanism which adopts the variable and huge traffic. The study also discusses how the mechanism will operate in two different channel conditions like multimode and single mode. The mechanism under multiple channel condition duplicates the throughput and performs the efficient operation as compared to the Single channel operation. Vincent et al. [15] have presented the concept of versatile MAC protocol that defines mobility handling issue of WSN. The model of VMAC combines the energy saving schedule based, and CBMAC protocol for short range of communication. In the work of Zhang et al. [16] application specific and semantic specific Hybrid MAC Protocol is discussed with Quality of Service (QoS) for WSN. Author has considered SNs as sinks of different applicability. The energy optimization and delay analysis results are analyzed which indicates better performance than other MAC protocols.

The combined work carried by Haiyang et al. [17] states mechanism with MAC protocol for WSN. The study analysis of the previous researches says the WSN have the issue of low batter power that needs to sort out. The study gives the some of the important MAC protocols in solving the WSN power issues. An integrated MAC protocol is given by Andrade et al. [18]. In this, WSN integration and radio over fiber (RoF) is performed by using MAC protocol. A very high throughput hybrid Mac protocol is presented in Jian et al. [19] for the millimeter wave WSN communication. The method significantly provides the energy efficiency in SN. This protocol consists of two networking elements like Relay master mote (RM), and Remote sensor mote (SM) and its state transitions are shown in Fig. 2.

In RM state transition once the state is initialized the RM will enter to Synchronization (Sync) that transfers a poll to build the connection among RM and SM. The SM will send an acknowledgment or ACK to the RM, and it will change its Idle state to Sleep state, which means no further synchronization is required. The scheduled data moves to the RM the state will move to Rx. Later the RM state will move to Tx State and assigns next data flow for SM to Idle. Then RM will enter the sleep state or else wait for next transition. Similarly, in next SM state transition (Right) will take place. The adaptive scheme of modulation for MAC with Fade state feedback quantization is presented by Kundu and Rajan [20]. In this one, a user will rotate the constellation without changing the transmit power that existing channel state, to meet the predefined minimum Euclidean distance at the destination. The performance and cost factors are improved than traditional methods.

The combined work for the Hybrid-MAC, Hybrid SensorMAC for Adhoc on multipath distance vector (AdMDC) is discussed in Kalaivaani and Rajeswari [21]. The study analysis presents that the AdMDC and H-MAC yield better performance results and energy optimization. An H-MAC protocol is presented in Hsieh et al. [22] for WSN. In this mechanism uses a cross-layer method which switches CSMA and TDMA over the network. The performance of this H-MAC is evaluated with different network density and achieved the improved energy efficiency and packet latency for WSN.

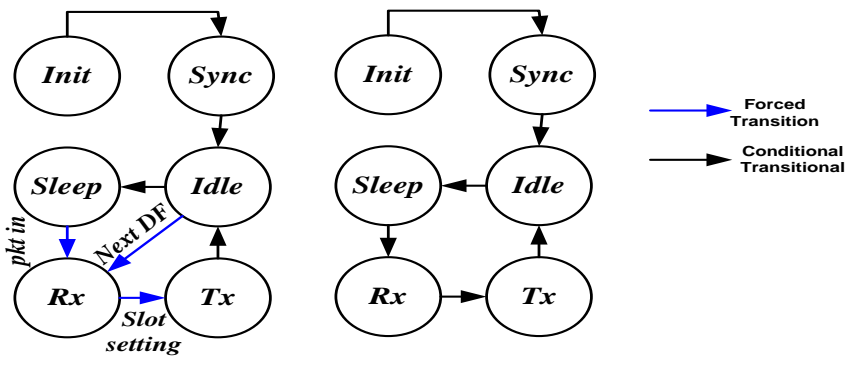

Fig. 2. State transition of RM (left) and SM (right). 
The aim for energy efficiency in WSN with MaC protocol is presented in Othman et al. [23] and QoS improvement. The outcomes from simulation and analytical are compared with QMAC protocol, and it was found that method proposed by the author has got more reliability and also low power consumption. The research performed by Wijetunge et al. [24] described the IEEE 802.15.4 based H-MAC protocol for hybrid WSNs monitoring. The protocol is defined to get the energy efficiency, low delay, and reliability in the monitoring of WSN. The objectives functions are optimized to meet the performance goal.

Author Shen et al. [25] presented MAC protocol to achieve energy efficiency. In this, the evaluation of various performance metrics is simulated with event simulator ns-2 mainly for the energy and benchmarked. The congestion control hybrid MAC mechanism for WSN is discussed in Priya and Manohar [26]. The protocol is the combination of both CSMA and TDMA, and that results in the advancement in the energy efficiency, packet delivery ratio, and delay performance. The conceptualize diagram of [26] is represented in Fig. 3 where the congestion takes place when data is transmitted. To minimize the congestion author have calculated the queue state and analyzed it.

$$
\text { QueueState }=\frac{\text { No.ofPkt } \sin \text { Queu. } 2^{3}}{\max \text { QueueSize }}
$$

Later the drop tail queuing systems are adopted in WSN. The packets of the congestion will drop at queue end. Hence the node with long queue will get affected than the small queue. The evaluation of channel allocation for H.mac (Multichannel) is described in Diab et al. [27]. In this simulation is performed for channel allocation efficiency of HMC-MAC and compared it with other methods. Later the interference rate is considered as the main metric, and results give how HMC-MAC will reduce the interference rate compared with other methods. Different consequences are considered with the same channel in 3, 2 and 1-hop neighborhood, to analyze the inference rate minimization concept.

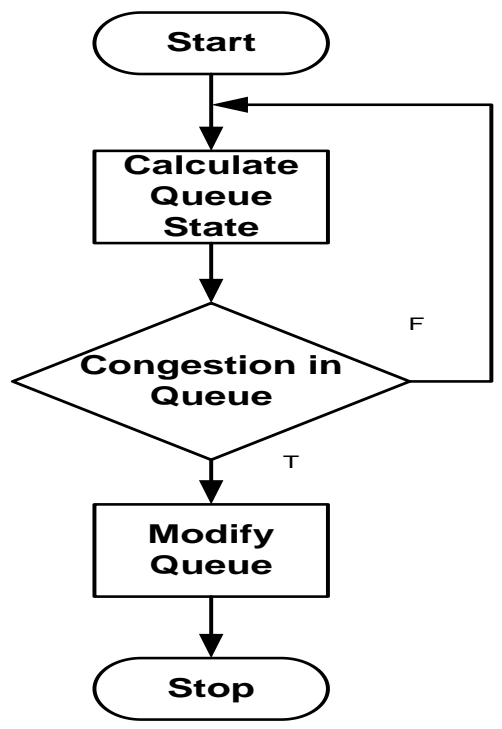

Fig. 3. Process diagram of [26].

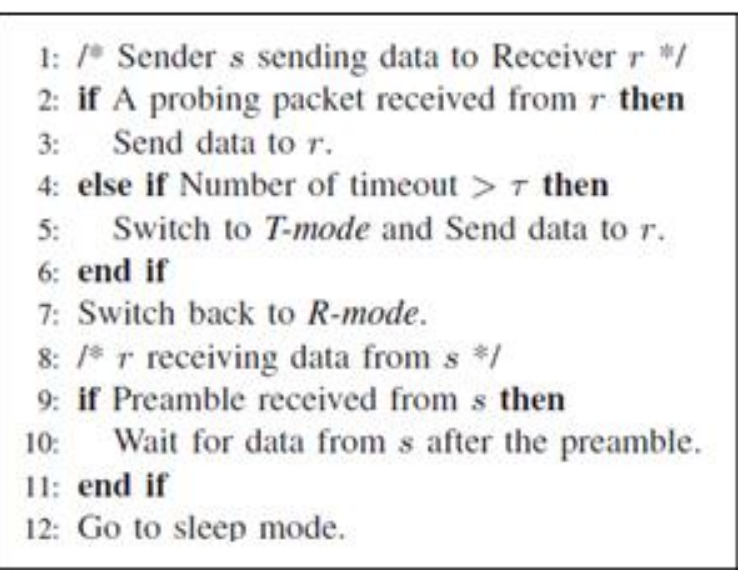

Fig. 4. Algorithm of [28] work.

The low powered duty cycle WSN is subjected to AsymMAC protocol with Asymmetric links in Won et al. [28]. The experimental results of the method yield high packet reception rate, low packet transmission delay than other protocol. The algorithm adapted for the method is given in Fig. 4. Where Tmode is transmission mode, $\mathrm{R}$-mode is receiving mode, $\tau$ is system throughput.

The characterization parameter in WSN is presented in Kadu and Deshpande [29]. The results exhibited from the grid topology, the average throughput achieved higher as compared to the other topologies like a chain and two random.

Author Kamal et al. [30] presented the secure/time synchronized and efficiency enhancement using the improved IHMAC. The outcomes are validated for enhanced efficiency. The performance and modeling analysis of MAP protocols with S-MAC and X-MAC are presented in Yang et al. [31]. In this Markov model together with the performance analysis gives estimated throughput, energy consumption, and delay for both synchronized and asynchronous duty-cycled MAC protocols. Akker et al. [32] described the interference effect among the heterogeneous WSN MAC protocol. The performance of TDMA, T-MAC other protocols is evaluated successfully. A dynamic de-centralized Hybrid MAC-Protocol is presented by Shah et al. [33]. In this, cognitive radios were used to solve spectrum scarcity problem for WSN applications. The outcome suggested that the mechanism is efficient, flexible and reliable for the real-time applications. Feng et al. [34] presented an approach to control overhead using S-MAC protocol.

\section{RESEARCH GAP}

All the explained MAC protocols exhibit both significance and limitation. Here, the problems which were not considered in many of past research works are discussed. Such unsolved problem poses a significant impediment towards the path of research work and hence we term it as a research gap.

- Less Applicability to the Real world: From the previous section, it has been seen that there are various MAC protocols with potential architectural design; however, it is still questionable that how much they are applicable in a real-world scenario. Any applications over realworld will always demand the compliance towards time 
and mission criticality. It is pretty significant that the data packets that are forwarded from the transmitting sensor should reach the receiver sensor before the time limit to ensure the time criticality as well the authentication of the true alarm notification. The various system calls for re-transmission which alternately schedules the data forwarding process is initiated to resist the data packet influence need to be dropped belongs to the slipped schedule. Thus, the data packet is required to be prioritized for transmission sequences. One common observation in existing technique is that it successfully maintain the timeliness, But, recently developed MAC schemes doesn't include a sequence of message transmission by the time limit. On the contrary, the algorithms are developed for minimizing the delay of data transfer. One of the unanswered questions found in all the existing techniques is that none of the techniques could explain the instantaneous sensor's response against the sudden event. For the MAC protocol to be working perfectly on real-time, it is required that they should have faster response time with lower delay (or latency), which is still unanswered.

- Doesn't solve energy problems completely: It is already known that the sensor node has a limited energy and battery lifetime. The limited lifetime also results in degradation of the network performance and causing intermittent (or un-stabilized links). As MAC protocols are mainly meant of energy conservation within the nodes, it is still questionable that how much of energy efficiency it can offer.

- More disadvantages compared to advantages: There are various MAC schemes that are using TDMA protocol in a WSN. The biggest challenges with such protocol usage are that its applicability is highly confined to specific traffic size. Till date, there is few research work which has proved optimal scalability achievement using TDMA based MAC protocols. Another flawed problem is the assumption that it makes in formulating MAC protocols. There are assumptions of the base station being covering entire sensors and compression to be existing only in higher layers. Such assumptions are completely unpractical. There is also a problem with event-driven schemes, which is large communication overhead as they mainly work on clustering principle. This degrades the performance of time synchronization that also negatively impacts on the network lifetime. More the surveillance area more is energy dissipation. A similar problem also exists for power efficient TDMA scheme. As they mainly depend on o clustering, so it results in overhead. The problem becomes worst in large-scale dynamic networks. The MAC techniques that use distributed schemes to conserve energy also suffer from issues e.g. delay which is generated from the node with lower residual energy. Not only do these existing schemes also have higher dependencies towards hardware capabilities to use non-interfering communication channels. Such schemes drastically minimize the data bit rate because of the split communication channel. Although TDMA schemes offer a better lifetime, its acceptance level is quite poor in WSN on scalability and its capability to function properly at the dynamic network.

- Fewer studies on Hybrid MAC schemes: A closer look at existing MAC schemes shows that almost every algorithm has advantages as well as disadvantages. Hence there are fewer attempts towards investigating about hybrid schemes. As these schemes integrate both CSMA and TDMA, such schemes can offer a greater deal of flexibility and energy efficiency. The studies [35]-[38], gives that there is a less supportability of the dynamic topology or mobility. The existing work on hybrid MAC schemes are also not found to evolve with a solution towards its inability to respond quickly to sudden event occurrence (in real-time).

Hence, a significant research gap is being explored in the area of MAC protocols in a WSN. The problems that are yet to be resolved are energy issues, scalability issues, delay and latency issues, overhead issues, and supports real time applications that will require being established with faster response time. This makes an obvious requirement to study and investigation the insights in related to these issues.

\section{FUTURE WORK}

The future work will be towards the direction of overcoming the research gap explored and briefed in the prior section. The tentative research works that can be carried out in future are briefed as follows:

- Effective integration of TDMA and CSMA scheme: The prime motive of this integration is to mainly achieve enhanced sustainable capacity towards increasing traffic load in WSN. To overcome the limitation of scalability and latency in the network, this integration scheme could focus on overcoming this issue. For this purpose, a specific control message could be designed with the inclusion of field for data priority. The scheme should also classify the time of communication into a uniform frames. It should be followed by an effective discovery of neighbor nodes to further assists in transmission, clustering, as well as synchronization. Better modeling could be achieved considering the state-transition of SYNC packets (i.e. RTS, CTS), etc. A robust modeling of search technique towards communication link could be designed to stabilize the energy dissipation and. The solution towards faster response time could be solved by energy modeling if the power allocation towards the sensor could be making more dynamic for precisely varying the transmittance power. A suitable balance between the crowded traffic scenario and packets to be delivered can be developed by focusing more on the packet prioritization. Such design could be suitably assessed using QoS as a performance factor.

- Architecture to support dynamicity: Architecture could be designed for ensuring minimal scalability, higher supportability of throughput, cutting short idle listening time. A unique discovery process using single hop could be designed which changes with the topology. A 
simply carried sensing model utilized for initialization of the communication process, and we encourage using only short preamble size to address overhearing problem. To solve maximum wake up time, we plan usage of involuntary buffering mechanism that can also contribute to enhancing the network lifetime. The next contribution could be to develop a synchronization algorithm to resist complexities of communication on different types of clusters. Even an empirical modeling can be carried out for energy modeling over presented MAC scheme using multiple energy distributions of the sensor node. Such scheme would minimize the idle listening time for the entire sensor, which will let the sensor go in hibernate mode if there is no data to be delivered.

- Decision-making model to meet peak traffic condition: Existing studies have less computational modeling on MAC protocols towards catering up the peak traffic demands in WSN. It is essential to identify the potential feature of forwarding rate by MAC protocols. Applying the approach using hybrid MAC protocols, this feature could be further enhanced. A clustering approach will be considered where all the nodes disperse in the cluster will possess mini-slots, which is accomplished by dividing the main time slot. The aggregator node performs carrier sense to join the network till base station or its neighbor node. In case the communication channel is failed to be received the aggregator node step over to the hibernation modes/stage and is allowed out of present state using an arbitrary period. An idea is not to miss any data as well as conserve energy also. Another advantage anticipated from this approach is effective synchronization policy as well as capability to meet the demands of meeting the peak traffic condition. For better scalability, we plan to develop discrete MAC protocols using a hybrid approach for both communications inside the cluster (member nodeaggregator node) and outside the cluster (aggregator node-aggregator node/base station).

\section{CONCLUSION}

This paper has discussed the usage of the MAC protocols and presented the most significant factors for network lifetime enhancement of the WSN. It also describes various forms of MAC schemes, along with the finding that most of the conventional MAC schemes are quite theoretically and fails to cater up to the need of optimality of the WSN performance. The paper, explains various schemes used for MAC protocol performance enhancement as well the research gap is extracted to set the future research direction. There is very less novelty in the existing approaches that causes very less improvement in network lifetime. Hence, our future work will look for continuing the investigation towards evolving up with new strategies and mathematical modeling to address such research gap.

\section{ACKNOWLEDGMENT}

The author gratefully acknowledges the support and encouragement of management and Dr. R Balakrishna, Principal, RajaRajeswari College of Engineering, Bengaluru.

\section{REFERENCES}

[1] K.J. Kim, N. Wattanapongsakorn, N. Joukov, "Mobile and Wireless Technologies," Springer Technology \& Engineering, pp. 252, 2016

[2] S. Khan, A-S. K. Pathan, N. A. Alrajeh, "Wireless Sensor Networks: Current Status and Future Trends," CRC Press, pp. 546, 2016

[3] K. J. Kim, N. Wattanapongsakorn, N. Joukov, "Mobile and Wireless Technologies," Springer, Technology \& Engineering, pp. 252, 2016

[4] M.S. Obaidat, S. Misra, "Principles of Wireless Sensor Networks," Cambridge University Press, pp. 433, 2014

[5] I. M. M. E. Emary, S. Ramakrishnan, "Wireless Sensor Networks: From Theory to Applications," CRC Press, pp. 799, 2013

[6] I. F. Akyildiz, M.C. Vuran, "Wireless Sensor Networks," John Wiley \& Sons, pp. 520, 2010

[7] Christophe J. Merlin, Wendi B. Heinzelman, "Duty Cycle Control for Low-Power-Listening MAC Protocols," IEEE Transactions on Mobile Computing, 2010

[8] F. Alfayez, M. Hammoudeh, A. Abuarqoub, "A Survey on MAC Protocols for Duty-cycled Wireless Sensor Networks," International Conference on Advanced Wireless Information and Communication Technologies (AWICT 2015), Vol. 73, pp. 482489,2015

[9] Joseph Kabara, M. Calle, "MAC Protocols Used by Wireless Sensor Networks and a General Method of Performance Evaluation," Computer Science, Information Systems, 2016

[10] S. I. A. Shah, M. llyas, H.T. Mouftah, "Pervasive Communications Handbook," CRC Press Technology \& Engineering, pp. 500, 2016

[11] D. Abdeli, S. Zelit and S. Moussaoui, "RTH-MAC: A real time hybrid MAC protocol for WSN," Programming and Systems (ISPS), 2013 11th International Symposium on, Algiers, pp. 153162,2013

[12] Q. Dong, W. Dargie and A. Schill, "Effects of sampling rate on collision probability in hybrid MAC protocols in WSN," 2010 IEEE Globecom Workshops, Miami, FL, pp. 213-218, 2010

[13] A. Karahan, İ Ertürk, S. Atmaca and S. Çakıcı, "Energy efficient hybrid MAC protocol for large scale wireless sensor networks," 23nd Signal Processing and Communications Applications Conference (SIU), Malatya, pp. 1549-1552, 2015

[14] S. Zhuo, Z. Wang, Y-Q. Song, Z. Wang, and L. Almeida, "A Traffic Adaptive Multi-channel MAC Protocol with Dynamic Slot Allocation for WSNs," IEEE Transactions on Mobile Computing, pp. 1536-1233, 2015

[15] V. Ngo, A. Anpalagan and I. Woungang, "Versatile medium access control (VMAC) protocol for mobile sensor networks," 7th International Wireless Communications and Mobile Computing Conference, Istanbul, pp. 836-841, 2011

[16] F.Zhang, F. Meng and Q.Xiong, "A novel application-semantics based hybrid QOS MAC in wireless sensor networks," Wireless Communications, Networking and Mobile Computing (WiCOM 2014), 10th International Conference on, Beijing, pp. 456-461, 2014

[17] H. Zhang, "Classic Efficient-Energy MAC Protocols for Wireless Sensor Networks," 6th International Conference on Wireless Communications Networking and Mobile Computing (WiCOM), Chengdu, pp. 1-4, 2010

[18] T. P. C. de Andrade, L. B. Oliveira, N. L. S. da Fonseca and O. C. Branquinho, "HMARS: A MAC protocol for integration of Radioover-Fiber and Wireless Sensor Networks," IEEE Third LatinAmerican Conference on Communications, Belem do Para, pp. 16, 2011

[19] W. Jian , C. I. Estevez, A. Chowdhury, Z. Jia, G-K. Chang, “ A Hybrid MAC Protocol Design for Energy-Efficient Very-HighThroughput Millimeter Wave Wireless Sensor Communication Networks," IEEE Third Latin-American Conference on Communications, 2010

[20] S. Kundu and B. S. Rajan, "An adaptive modulation scheme for two-user fading MAC with quantized fade state feedback," IEEE 23rd International Symposium on Personal, Indoor and Mobile Radio Communications - (PIMRC), Sydney, NSW, pp. 512-518, 2012 
[21] P. T. Kalaivaani and A. Rajeswari, "An analysis of H-MAC, HSMAC and H-MAC based AOMDV for wireless sensor networks to achieve energy efficiency using spatial correlation concept," Electronics and Communication Systems (ICECS), 2nd International Conference on, Coimbatore, pp. 796-801, 2015

[22] T. H. Hsieh, K. Y. Lin and P. C. Wang, "A hybrid MAC protocol for wireless sensor networks," Networking, Sensing and Control (ICNSC), 2015 IEEE 12th International Conference on, Taipei, pp. 93-98, 2015

[23] J. Ben-Othman, L. Mokdad and B. Yahya, "An Energy Efficient Priority-Based QoS MAC Protocol for Wireless Sensor Networks," 2011 IEEE International Conference on Communications (ICC), Kyoto, pp. 1-6, 2011

[24] S. Wijetunge, U. Gunawardana and R. Liyanapathirana, "IEEE 802.15.4 based hybrid MAC protocol for hybrid monitoring WSNs," Local Computer Networks (LCN), IEEE 38th Conference on, Sydney, NSW, pp. 707-710, 2013

[25] J. Shen, F. Yi, S. Moh and I. Chung, "Energy Efficiency of MAC Protocols in Wireless Sensor Networks," 2011 International Conference on Information Science and Applications, Jeju Island, pp. 1-10, 2011

[26] B.Priya, S.Solai Manohar, " CH-Mac: Congestion Control Hybrid MAC for Wireless Sensor Network," 4th ICCCNT, 2013

[27] Rana Diab, G'erard Chalhoub and Michel Misson, "Hybrid MultiChannel MAC Protocol for Wireless Sensor Networks: Interference Rate Evaluation," IEEE International Conference on Communications, 2013

[28] M.Won, T. Park, and S. H. Son, "Asym-MAC: A MAC Protocol for Low-Power Duty-Cycled Wireless Sensor Networks with Asymmetric Links," IEEE Communications Letters, Vol. 18, No. 5,2014

[29] S.D. Kadu, V.S. Deshpande, "Characterization of Throughput in Wireless Sensor Network for MAC and Routing Protocol," International Conference on Cloud \& Ubiquitous Computing \& Emerging Technologies, 2013
[30] P. Kamal, S. Singh, Z. M. Livinsa, "Secure Time Synchronization and Efficiency Enhancement using Improved IHMAC," IEEE Sponsored 2nd International Conference on Innovations in Information Embedded and Communication Systems ICIIECS, 2015

[31] O.Yang, and W. B. Heinzelman, "Modeling and Performance Analysis for Duty-Cycled MAC Protocols with Applications to SMAC and X-MAC," IEEE Transactions on Mobile Computing, Vol. 11, No. 6, 2012

[32] D. V. D. Akker, B. Braem, C. Blondia, "On the Effects of Interference between heterogeneous Sensor Network MAC Protocols," Eighth IEEE International Conference on Mobile AdHoc and Sensor Systems, 2011

[33] M. A. Shah, Ghazanfar A. Safdar, C. Maple, "DDH-MAC: A Novel Dynamic De-Centralized Hybrid MAC Protocol for Cognitive Radio Networks," 2011

[34] H. Feng, L. Ma, S. Leng, "A Low Overhead Wireless Sensor Networks MAC protocol," IEEE International Conference, 2010

[35] B. Shrestha, K. W. Choi and E. Hossain, "A Dynamic Time Slot Allocation Scheme for Hybrid CSMA/TDMA MAC Protocol," in IEEE Wireless Communications Letters, Vol. 2, No. 5, pp. 535$538,2013$.

[36] V. Nguyen, T. Z. Oo, P. Chuan and C. S. Hong, "An Efficient Time Slot Acquisition on the Hybrid TDMA/CSMA Multichannel MAC in VANETs," in IEEE Communications Letters, Vol. 20, No. 5, pp. 970-973, 2016.

[37] Y. Xu, P. Xu, W. Zeng and Q. Tian, "Hybrid MAC based resource management scheme for kiosk service in 802.15.3c WPAN," 7th International Wireless Communications and Mobile Computing Conference, Istanbul, pp. 516-521, 2011

[38] R. Zhang, L. Cai and J. Pan, "Performance Study of Hybrid MAC Using Soft Reservation for Wireless Networks," IEEE International Conference on Communications (ICC), Kyoto, pp. 15,2011 